\title{
A structured light method for underwater surface reconstruction
}

\author{
Amin Sarafraz ${ }^{\mathrm{a}}$, Brian K. Haus ${ }^{\mathrm{b}}$ \\ ${ }^{a}$ Department of Civil, Architectural and Environmental Engineering, University of Miami \\ ${ }^{b}$ Rosenstiel School of Marine and Atmospheric Science, University of Miami
}

\begin{abstract}
A new structured-light method for 3D imaging has been developed which can simultaneously estimate both the geometric shape of the water surface and the geometric shape of underwater objects. The method requires only a single image and thus can be applied to dynamic as well as static scenes. Experimental results show the utility of this method in non-invasive underwater $3 \mathrm{D}$ reconstruction applications. The performance of the new method is studied through a sensitivity analysis for different parameters of the suggested method.
\end{abstract}

Keywords: Water surface estimation, 3D reconstruction, Structured light.

\section{Introduction}

Three dimensional surface imaging has been an active area of research for decades and was reached a stage of maturity, thanks to digital cameras and ever-increasing computational power. Applications of 3D surface imaging range from traditional mapping applications, structural monitoring, cultural heritage documentation, quantifying landform change to forensic, medical, biology and many other fields.

There are different methodologies for 3D reconstruction using optical cameras, such as stereo and multi camera imaging, photometric stereo, structured light and shape from $\mathrm{X}$ techniques, to name a few. Researchers have extended some of these methods to adapt to the underwater environment. Examples of such pioneering studies in underwater photogrammetry include $[1,2,3,4]$. Also there are several books and studies $[5,6,7]$ that discuss the pros and cons of each methodology, where the same pros and cons are still valid for underwater 3D reconstruction.

Preprint submitted to ISPRS Journal of Photogrammetry and Remote SensingJanuary 14, 2016

(C) 2016. This manuscript version is made available under the Elsevier user license

http://www.elsevier.com/open-access/userlicense/1.0/ 
In this paper our focus is on extending structured light techniques in a specific case of a two phase environment where the camera is underwater and the projector is above water. Structured light techniques simplify the problem of $3 \mathrm{D}$ reconstruction with the help of controlled illumination. They basically consist of acquiring $2 \mathrm{D}$ image(s) of the scene while illuminating the scene with spatially varying intensity pattern(s). The nonplanar geometric shape of the surface distorts the projected structured-light pattern as seen from the camera. Then the information from the distortion of the projected structured-light pattern is used to extract the 3D surface shape.

Numerous structured light techniques for 3D surface imaging are currently available. The techniques that have been developed range from those that require multiple images (multi-shot techniques) $[8,9,10,11,3]$ to reconstruct a surface to those that require only a single image (single-shot techniques) $[12,13,14,15,16,17]$. Multiple-shot techniques can be used in cases where the target is static and the application does not impose any constraint on the acquisition time. Generally, multiple-shot techniques may often result in a more reliable and more accurate $3 \mathrm{D}$ reconstructed surface. Because of this, if the target is static, multiple-shot techniques are recommended. However, if the target is moving, using single-shot techniques is required. A more detailed overview of available structured light techniques can be found in $[18,19]$.

In all of above-mentioned structured light techniques, the assumption is that the projector and the camera are in the same (homogeneous) medium, e.g. both camera and projector are placed underwater or in an open-air environment. However, there may be applications that require the projector and the camera to be in different mediums or in which the light must pass through an inhomogeneity. For example, consider air-sea interface studies that need simultaneous estimation of water surface and sea floor in shallow waters. Subsurface inspection of harbor facilities, bridges or vessels may also be enabled by illumination or imaging from outside the water. In this paper, we seek to develop a structured-light technique that employs a projector above water and an underwater camera for estimation of both the water surface and sea floor. In this case, the water surface is dynamic and changes instantaneously. Because of dealing with a dynamic surface, the method has to use a single image. A unique color pattern was generated from a projector located above the water and images were acquired with a camera located under water. With this unique color pattern only one image was required to reconstruct both the water surface and the sea floor. 


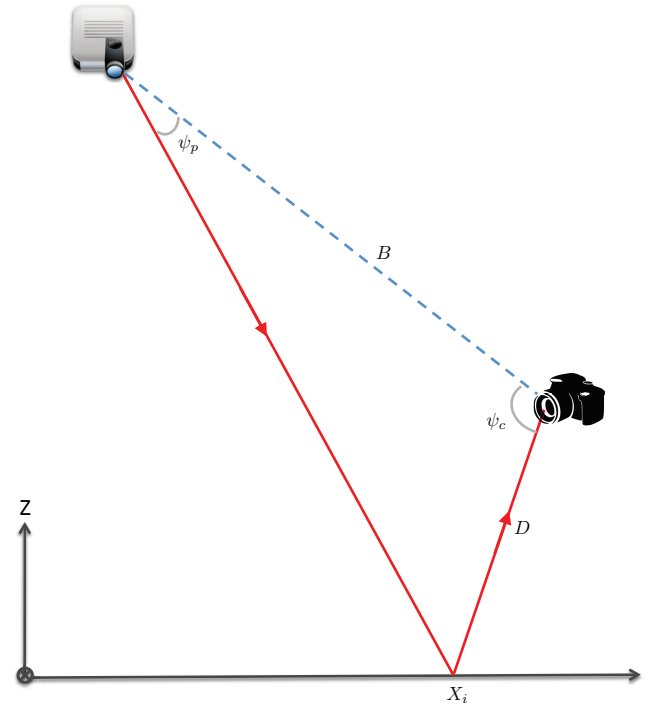

(a)

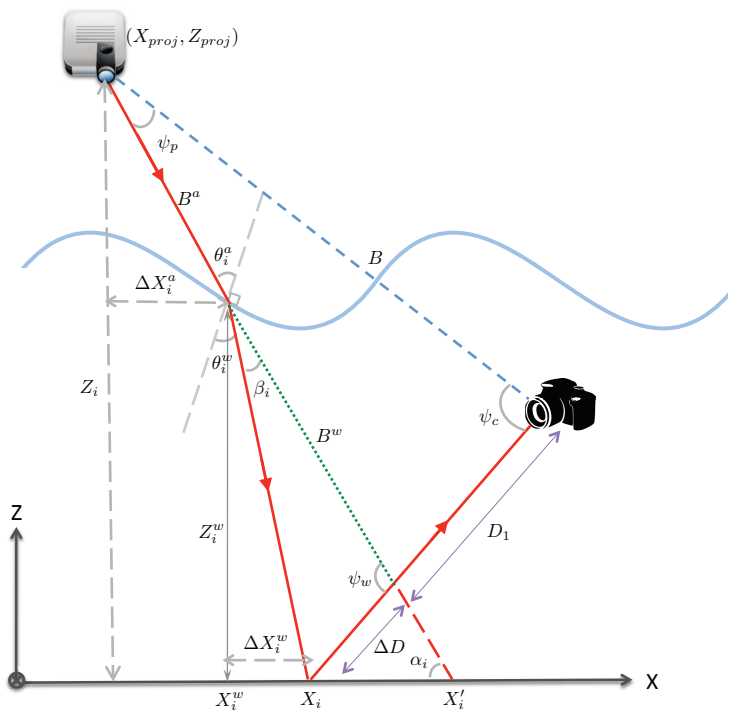

(b)

Figure 1: Orthogonal projection of a 3D ray in $X Z$ plane in a projector-camera system: (a) both camera and the projector are in the same medium, (b) camera and the projector are in different medium, in this particular case the projector is above water and the camera is underwater. The ray refracts after hitting the water surface based on its incidence angle $\theta_{i}^{a}$.

\section{2. problem statement}

Consider a light ray emitted from the projector and represented by a vector $\mathbf{v} \in \mathbf{R}^{3}$. Let $S$ be a nontrivial subspace of a vector space $\mathbf{R}^{3}$ and assume that $\mathbf{v}$ is a vector in $\mathbf{R}^{3}$ that does not lie in $S$. Then the vector $\mathbf{v}$ can be uniquely written as a sum of its orthogonal projections $\mathbf{v}_{\| S}+\mathbf{v}_{\perp S}$, where $\mathbf{v}_{\| S}$ is parallel to $S$ and $\mathbf{v}_{\perp S}$ is orthogonal to $S$ [20]. The vector $\mathbf{v}_{\| S}$, which actually lies in $S$, is called the projection of $\mathbf{v}$ onto $S$. If $\left(\mathbf{v}_{1}, \mathbf{v}_{2}\right)$ form an orthogonal basis for $S$, then the projection of $\mathbf{v}$ onto $S$ is the sum of the projections of $\mathbf{v}$ onto the individual orthogonal basis vectors [20]. Thus, a 3D light ray can be represented by its orthogonal projections on $X Z$ and $Y Z$ planes. For illustration purposes, we only show orthogonal projections of light rays on $X Z$ plane in Fig. 1.

Consider a typical structured-light system in which both camera and the projector are in the same medium, see Fig. 1(a). A projector illuminates the object with a spatially varying structured light and an imaging sensor is used to acquire a $2 \mathrm{D}$ image of the scene. In this case, the projected structured-light pattern distorts solely due to the geometric shape of the object surface. As shown in Fig. 1(a), the geometric relationship between an imaging sensor, a structured-light projector, and an object surface point can be expressed by 


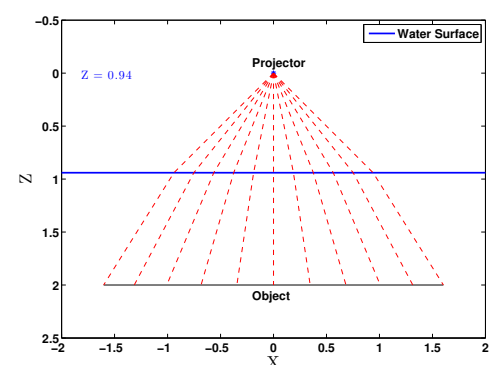

(a)

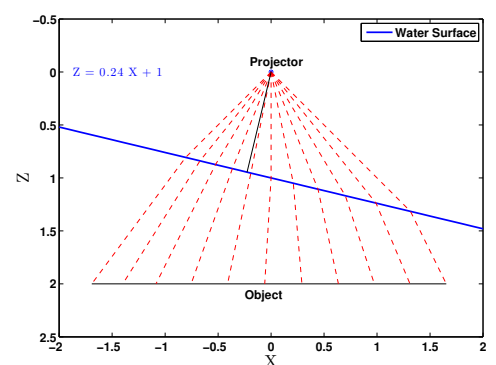

(b)

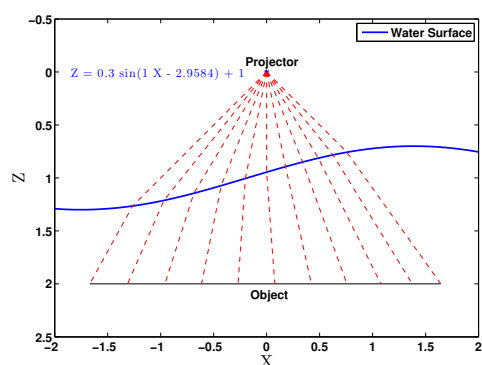

(c)

Figure 2: Orthogonal projection of the ray refraction after hitting water surface with a (a) flat surface with Height $=0.94 \mathrm{~m}$, (b) flat surface with Slope $=0.24$ and Intercept 1, (c) water surface model based on linear small amplitude wave theory [21]

the triangulation principle as

$$
D=B \frac{\sin \left(\psi_{p}\right)}{\sin \left(\psi_{c}+\psi_{p}\right)}
$$

Now consider the scene in Fig. 1(b) where the projector is located above water and the camera is under water. In this case, the distortion of the pattern is due to both the geometric shape of the water surface and the geometric shape of the object surface. To recover the object surface, one should consider distortions from the water surface. Note that, the path of light ray in this case has two parts; one above water and one under water. Both parts are 3D rays and their orthogonal projections on $X Z$ plane are shown in Fig. 1(b). While a real water surface inherently contains variability in both the $X$ and $Y$ directions, for simplicity here we will consider the $X$-axis lying in the wave propagation direction and the $Y$-axis which is orthogonal to it separately, as is commonly implemented in Airy wave theory [21]

To demonstrate the effect of water surface shape on the projected pattern, consider the scene in Fig. 2 where a projector illuminates an underwater flat object while its line of sight is perpendicular to the object's surface. These plots show the effect of refraction of the projected rays when they enter the water. Fig. 2(a), (b), and (c) illustrate the behavior of the rays against a flat, sloped, and sinusoidal water surfaces, respectively. As can be seen, each ray has a different refraction behavior based on its incidence angle. When a light ray passes through the water surface, Snell's law can describe the relationship between the angles of incidence and refraction of that ray, i.e.

$$
n_{w} \sin \left(\theta_{i}^{w}\right)=n_{a} \sin \left(\theta_{i}^{a}\right)
$$

where $n_{a}$ represents the refractive index of the air and $n_{w}$ is the refractive index of the water. Also, $\theta_{i}^{a}$ and $\theta_{i}^{w}$ respectively represent the incidence angle and the refraction angle of the $i^{\text {th }}$ ray. 
Because of the refraction effect, a unique projected pattern will have different shapes, even on a flat object at the same distance from the projector, depending on the water surface shape. If one can estimate the water surface, then it is possible to predict the path of each projected ray and account for the refraction effect on the distortion of the projected pattern on the underwater object. The method used to estimate the water surface is discussed in section 3.3 .

In order to estimate the geometric shape of the water surface and underwater objects, the camera-projector system must first be calibrated. In the next section, the details of the camera-projector (system) calibration will be explained.

\section{Method}

\subsection{System calibration}

Calibration is an essential part of a camera-projector system. This includes intrinsic calibration of both the camera and the projector as well as extrinsic calibration of the camera-projector system. The accuracy of the calibration step plays a critical role in the measurement accuracy of the camera-projector system.

The topic of calibration of optical cameras have been extensively studied in both computer vision and photogrammetry communities, which resulted in an extensive body of literature $[22,23,24,25,26,27,28,29,30]$ over the past couple of decades and it still remains a topic of research interest. Each method has its own pros and cons and there are several studies [31, 32] that compare different calibration methods.

For camera calibration the method proposed by Zhang [29, 33] and implemented by Jean-Yves Bouguet [34] was adopted here. There are advanced calibration techniques available which can obtain higher accuracy, as is reported in [31]. However, to the best of our knowledge there is no freely available code for such techniques and they are difficult to implement in an underwater environment due to the requirement for special calibration laboratories.

The Zhang method [29, 33] uses a pinhole camera model and requires images at several angles and distances of a known calibration object. The intrinsic parameters in this approach are focal length, principal point, skew coefficient, and distortions. For a finer scale reconstruction the use of more 
accurate calibration methods is recommended but for the purpose of exploration of our proposed method, Zhang's calibration algorithm is sufficient.

A projector can be considered as an inverse camera and can be calibrated using camera calibration algorithms. A pre-calibrated camera and a calibration plane can assist in establishing the correspondence between the projector and the world coordinate system. By taking images of a checkerboard on a plane, the correspondences between the calibrated camera and the plane can be established. Then through ray-plane intersection, the coordinates of any given point in the camera that falls onto this plane could be transferred to the plane coordinate system. Therefore, if the projector emits a ray or a pattern that the camera can locate, its intersection with the plane can be translated into the corresponding 3D world point. Then the projector can be calibrated using the Zhang based calibration method that was used to calibrate the camera. For more details of camera-projector calibration see $[35,36]$.

\subsection{Projected pattern}

Since in this application the water surface is dynamic, a structure for the illuminant that enables the localization of all light spots in a single-shot was required. The pattern proposed in $[13,37]$ was adopted. These approaches incorporated an algorithm to generate an array that preserves the uniqueness of subwindows. A subwindow of size $n \times n$ is a set of $n \times n$ adjacent color spots within the pattern that form a unique color combination. Thus every color spot in the pattern could be easily identified by its surrounding subwindow. Our method is designed to work in pure (salt or fresh) water and clean ocean and it implicitly assumes that the absorption and scattering should be small enough that the projected pattern can be seen. We use three colors (red, green, blue) to create the pattern but there is no restriction on the number of colors and the type of colors that could be used in order to create such pattern. However, increasing the number of colors potentially increases the noise sensitivity when measuring colorful scenes.

The depth per pixel used in the pattern is also an important parameter. The noisier the application environment, the smaller the number of colors used should be. When increasing the number of colors, differentiating between the colors becomes more difficult. Therefore, the three color combinations are the most robust against noise. Note that two colors could not create a large enough unique number of subwindows in a projected pattern. 


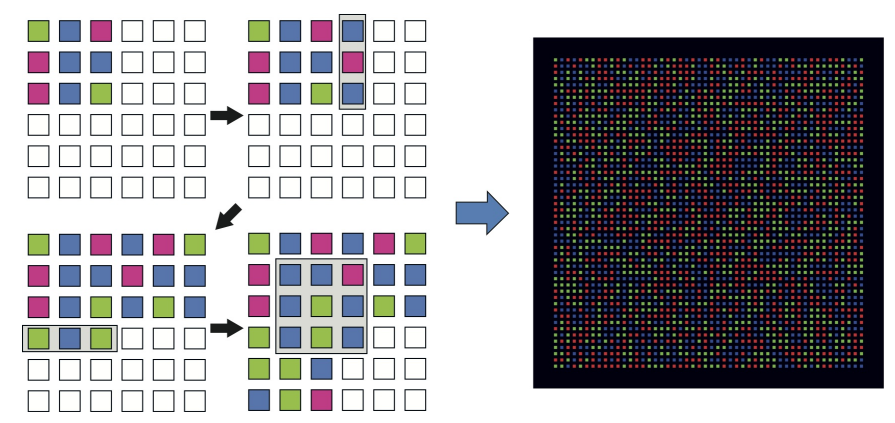

Figure 3: Generating pattern with $3 \times 3$ subwindows using three colors $(R, G, B)$ : (left) step by step pattern generation for a $6 \times 6$ array (right) example of a generated $50 \times 50$ pattern.

Also, choosing colors with higher contrast among each other and against the underwater object will increase the chance of their detection in the images.

\subsection{Water surface estimation}

We assume that the water surface can be represented by a parametric model (e.g. a polynomial) and the model components in $X$ and $Y$ directions are independent, so the model parameters in each direction can be estimated separately. Since there are no limits on the number of parameters, the water surface can have any complex shape. Consider the scene in Fig. 1(b). Without loss of generality, the water surface along only the $X Z$ plane was considered. A projector was located above the water and a camera was located under water. It is assumed that both camera and the projector are calibrated, so the transformation from camera frame to projector frame is established. The water surface is a dynamic surface. A light ray emitted from the projector passes through the water surface and refracts based on Snell's law. Then it reflects from the object surface towards the camera. If the shape of the water surface is known, one can trace each ray and predict the coordinates of that ray in the projector coordinate frame. Using the predicted and observed coordinates of $n$ number of rays, the problem of water surface estimation can be solved as an optimization problem. In other words, we need to find a set of water surface parameters a that minimizes the distance between predicted coordinates of rays and their observed coordinates:

$$
\underset{\mathbf{a}}{\operatorname{minimize}} J(\mathbf{a})
$$


where $J(\mathbf{a})$ is the cost function and is equal to

$$
J(\mathbf{a})=\frac{1}{2 n} \sum_{i=0}^{n}\left(X_{i}-X_{i}^{p}\right)^{2}
$$

In Eq.(3) and Eq.(4), $n$ is the number of rays that will participate in water surface estimation, $X_{i}$ is the observed coordinate of ray $i$ and $X_{i}^{p}$ is the predicted coordinates of ray $i$. Note that $n$ should not be less than the number of parameters that describe the water surface. The exhaustive search algorithm was used as a global optimization technique to solve this problem. Other optimization techniques can be used to find the water surface parameters as long as they do not entrap in local minima. Similarly, the water surface in $Y$ direction can be estimated using $Y$ coordinates of $n$ rays.

To obtain the predicted and observed coordinates, we use a plane with a checkerboard attached to it (see Fig. 4). Using a calibrated camera, the rotation and translation matrices of any plane can be obtained. Consider a plane with an attached checkerboard (calibration grid) and concentrate on the camera reference frame attached to that grid. Fig. 4 shows the reference frame $(O, X, Y, Z)$ attached to that calibration grid.

Let $P$ be a point with coordinate vector $\mathbf{X}_{p}=\left(X_{p}, Y_{p}, Z_{p}\right)$ in the grid reference frame shown in Fig. 4 . Let $\mathbf{X}_{p}^{c}=\left(X_{p}^{c}, Y_{p}^{c}, Z_{p}^{c}\right)$ be the coordinate vector of $P$ in the camera reference frame. Then $\mathbf{X}_{p}$ and $\mathbf{X}_{p}^{c}$ are related to each other through the following rigid motion equation:

$$
\mathbf{X}_{p}^{c}=R^{c} \mathbf{X}_{p}+T^{c}
$$

In particular, the translation vector $T^{c}$ is the coordinate vector of the origin of the grid pattern $(O)$ in the camera reference frame, and the third column of the matrix $R^{c}$ is the surface normal vector of the plane containing the planar grid in the camera reference frame.

The translation vector $T^{c}$ and the rotation matrix $R^{c}$ of the plane can be recovered in the camera reference frame using corresponding points. For this purpose, one can use the corners of the checkerboard. These corners can be determined in the image plane. Once the coordinates of a point are determined in the image plane, they can be expressed in the camera reference frame using the intrinsic camera parameters. On the other hand, each corner on the calibration board becomes $\mathbf{X}_{p}=\left(X_{p}, Y_{p}, 0\right)$. By plugging corresponding $\mathbf{X}_{p}$ 's and $\mathbf{X}_{p}^{c}$ 's into Eq. (5), the $T^{c}$ and $R^{c}$ will be estimated. The 


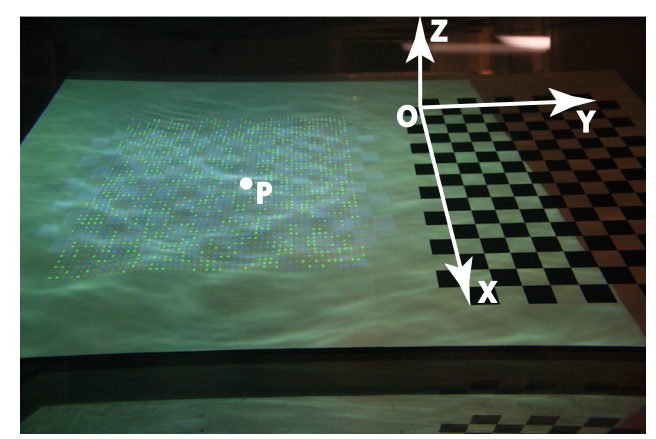

Figure 4: Calibrated camera image of a subsurface scene under a wavy interface including the projected image and a checkerboard attached to the white board. Object coordinate system shown in white. The size of each square on the checkerboard is 1 inch by 1 inch.

problem is equivalent to homography computation and different solutions are discussed in [38], as well as degenerate configurations that should be avoided. Some solutions can lead to unstable results because of the numerical calculation, inaccurate point correspondences and so on. We first compute an initial guess for the homography through the quasi-linear method. Then, if the total number of non collinear points is larger than 4, we optimize the solution by minimizing the reprojection error [34]. Once the transformation from the plane coordinates system to camera reference frame is established, the coordinates of any point on the plane can be determined in the camera reference frame.

Using the rotation matrix and translation vector between the camera and the projector obtained from the camera-projector calibration, one can transform object points from the camera reference frame to the projector reference frame. The coordinates of the pattern in the projector image plane can be expressed in the projector reference frame using the intrinsic projector parameters obtained from the camera-projector calibration.

Suppose that the parametric representation of the water surface function in the projector reference frame is known; i.e. $Z^{w}=F\left(X^{w}, \mathbf{a}\right)$ where $\mathbf{a}=$ $\left\{a_{0}, a_{1}, \ldots, a_{n}\right\}$ are parameters of the function. The intersection of ray $i$ and the water surface is represented by $\left(X_{i}^{w}, Z_{i}^{w}\right)$. See Fig. 1(b) for illustration of these parameters. This intersection can be determined by finding a point that satisfies both the water surface function and the line that represents the ray from the projector:

$$
Z_{i}^{w}=Z_{p r o j}+\frac{\left(Z_{i}^{\prime}-Z_{p r o j}\right)\left(X_{i}^{w}-X_{p r o j}\right)}{\left(X_{i}^{\prime}-X_{p r o j}\right)}
$$

Where $\left(X_{\text {proj }}, Z_{\text {proj }}\right)$ are the projector coordinates. According to Fig. 1(b), one can write $X_{i}$ as:

$$
X_{i}=X_{p r o j}+\Delta X_{i}^{a}+\Delta X_{i}^{w}
$$


where

$$
\Delta X_{i}^{a}= \pm\left|\left(Z_{i}^{w}-Z_{\text {proj }}\right) \cot \left(\alpha_{i}\right)\right|
$$

and

$$
\Delta X_{i}^{w}= \pm\left|\left(Z_{i}^{w}-Z_{i}\right) \cot \left(\alpha_{i}+\beta_{i}\right)\right|
$$

In Eq.(8) and Eq.(9), $\alpha_{i}$ can be estimated using the intrinsic parameters of the projector which have been already estimated in the calibration step, i.e. $\tan \left(\alpha_{i}\right)=f_{\text {proj }} / x_{i}^{p}$ where $f_{\text {proj }}$ is the focal length of the projector. Also, $\beta_{i}$ is the difference between the incident angle $\theta_{i}^{a}$ and the refractive angle $\theta_{i}^{w}$ :

$$
\beta_{i}=\theta_{i}^{a}-\theta_{i}^{w}
$$

Note that all angles are in the $X Z$ plane. The incident angle for each ray can be calculated by the following equation:

$$
\theta_{i}^{a}=\frac{\pi}{2}-\left(\alpha_{i}-\gamma_{i}\right)
$$

where $\gamma_{i}$ is the slope of the water surface at the intersection of the ray $i$ with the water surface. If the water surface is known, it can be calculated by

$$
\tan \left(\gamma_{i}\right)=\frac{d F\left(X_{i}^{w}, \mathbf{a}\right)}{d X_{i}}
$$

In order to compute the refractive angle $\theta_{i}^{w}$ in the $X Z$ plane, in general one can not simply apply Snell's law. Instead the refractive angle can be calculated using the following equation (see Appendix A for the details)

$$
\theta_{i}^{w}=\sin ^{-1}\left(\frac{\sin \left(\theta_{i}^{a}\right)}{\eta^{\prime}}\right)
$$

where $\eta^{\prime}=\sqrt{\eta^{2}-\sin ^{2}(\zeta)} / \cos (\zeta), \eta=n_{w} / n_{a}$, and $\zeta$ is the angle between the incident ray and the $X Z$ plane. There are always zones in the footprint of the projected pattern that $\eta$ and $\eta^{\prime}$ could be used interchangeably depending on what percentage of error could be tolerated in an application. In those zones the magnitude of error between using $\eta$ instead of $\eta^{\prime}$ for calculating the $X$ coordinate is negligible, thus one can replace $\eta^{\prime}$ with $\eta$ in the Snell's law

$$
\theta_{i}^{w}=\sin ^{-1}\left(\frac{\sin \left(\theta_{i}^{a}\right)}{\eta}\right)
$$

Figure 5 shows the magnitude of error in percentage for different combinations of $\theta^{a}$ and $\zeta$. Note that the incidence angles greater than 45 degrees 


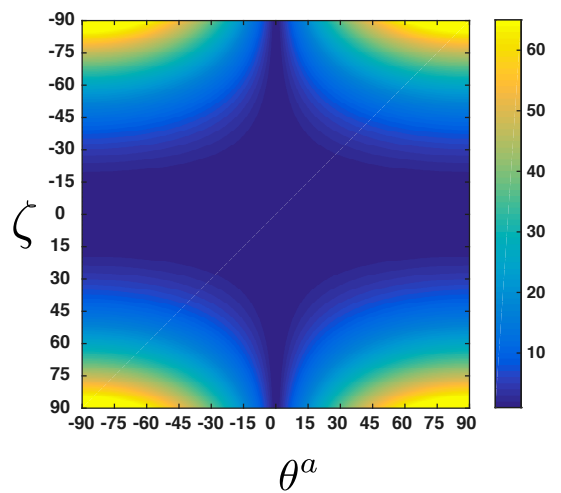

(a)

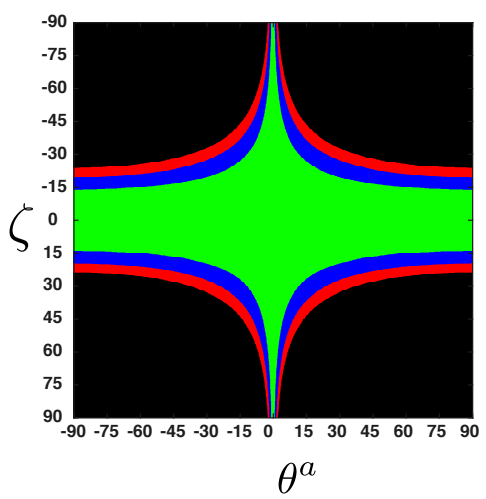

(b)

Figure 5: Magnitude of error in using $\eta$ instead of $\eta^{\prime}$ in calculating $X$ coordinates for each point (a) for all values of incidence angle $\theta^{a}$ and the angle between the incident ray and the $X Z$ plane $\zeta$ (b) green: error $\leq 1 \%$, blue: $1 \%<$ error $\leq 2 \%$, red: $2 \%<$ error $\leq 3 \%$, black: error $>3 \%$. Values of error are in percentage and values of $\theta^{a}$ and $\zeta$ are in degrees.

are extreme cases and rarely happen in studies similar to our study. To put this in perspective, in all our experiments, the horizontal field of view of the projector was $\sim 37$ degrees, which translates into $\sim 18.5$ degrees for the maximum value for the incidence angle on a flat water surface. Our projected pattern only covered partial field of view of the projector in which the maximum value for the incidence angle on a flat water surface was $~ 10$ degrees. We have used only the rays that fall within the $1 \%$ of error margin (the green area in Figure 5) for water surface estimation and object reconstruction.

\subsection{Object reconstruction}

Once the water surface parameters are determined, the path of each ray passing through the water surface can be predicted. When both the camera and the projector are in the same medium, in order to compute object coordinates, only one distance, $D$, needs to be determined (Fig. 1(a)). This can be done using Eq.(1). However, in this system, because of refraction of the light rays, this equation is not valid for computing the distance between the camera and the object. In the presence of the refracted light rays, the distance between the camera and the object, can be computed by adding or subtracting $\Delta D$ from $D$ as shown in Fig. 1(b).

$$
D=B \frac{\sin \left(\psi_{p}\right)}{\sin \left(\psi_{c}+\psi_{p}\right)} \pm \Delta D
$$

in Eq.(15), if the ray refracts towards the camera, use $-\Delta D$, and if it refracts away from the camera, use $+\Delta D$. 


$$
\Delta D=B^{w} \frac{\sin \left(\beta_{i}\right)}{\sin \left(\beta_{i}+\psi_{w}\right)}
$$

$B^{w}$ is the distance between two intersections: (1) the intersection of the ray from projector and the water surface and (2) the intersection of the undeviated ray from projector and the reflected ray from object towards the camera. By the water surface function obtained using the procedure in Section 3.3, one can obtain its intersection with the ray projected from the projector. Also, using $D$ calculated by Eq.(1), the coordinates of the camera, and the direction of the ray reflected from the object, the second intersection can be obtained.

\section{Experiments and results}

Several experiments were conducted to explore the ability of the proposed structured light method to estimate the water surface and the underwater object shape. The results are presented here for both synthetic and real data. A cylindrical object with a flat top and a spherical object were used for 3D reconstruction. These examples demonstrate that the method works for objects with different shapes. The experiments were conducted in the presence of both flat and wavy water surfaces. The flat water surface was represented with a linear model and the wavy water surface with a sinusoidal model using linear small amplitude wave theory [21, 39] . Other parametric models could be used if they are more appropriate for a particular condition of the water surface.

\subsection{Synthetic data}

To evaluate the correctness and performance of the method, synthetic data was created for both linear and sinusoidal water surfaces (see Fig. 2 (b),(c)). The world coordinate origin was assumed to be at the projector center. All internal calibration parameters for the projector were known and were set to $\left(x_{c}^{\text {Proj }}, y_{c}^{\text {Proj }}\right)=(0,0), f_{\text {Proj }}=100$ pixel, and no lens distortion for simplicity. With known water surface parameters, the $(X, Z)$ pairs for each ray were calculated using Eq.(6) to Eq.(9) .

In the first synthetic dataset, it was assumed that the water surface has a linear function, i.e. $Z_{i}^{w}=a_{1} X_{i}^{w}+a_{2}$. In this dataset, $a_{1}$ and $a_{2}$ were set to -0.07 and 1.16 respectively. In other words, it was assumed that the projector was located $1.16 \mathrm{~m}$ above a water surface with slope -0.07 . The 


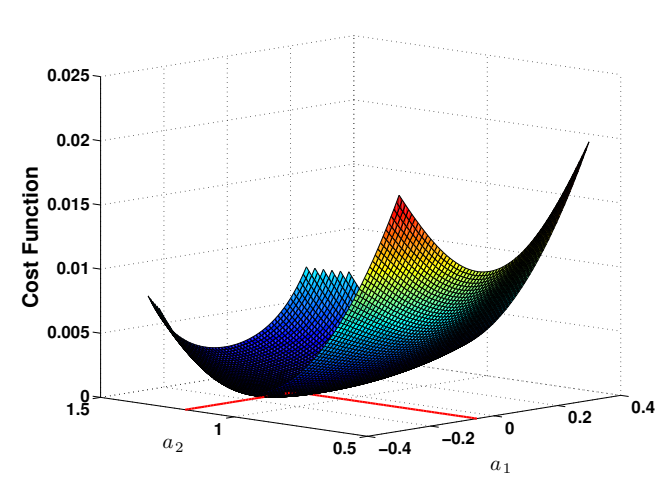

(a)

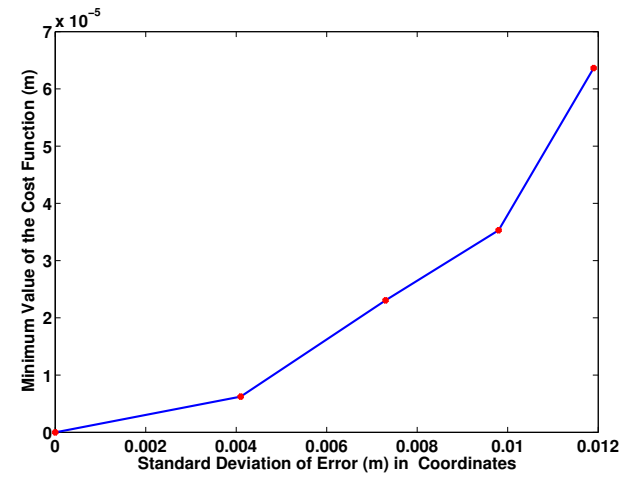

(b)

Figure 6: (a) Cost function for a synthetic flat water surface of the form $Z_{i}^{w}=a_{1} X_{i}^{w}+a_{2}$. The $a_{1}$ and $a_{2}$ were set to -0.07 and 1.16. Standard deviation of added random noise to coordinates was equal to $0.0113 \mathrm{~m}$. (b) Minimum value of the cost function for estimating flat water surface parameters while adding different levels of noise to the actual coordinates of 11 rays. As the level of noise in the input data increases, the minimum value of the cost function increases.

$(X, Z)$ pairs for 11 rays were calculated using Eq.(6) to Eq.(9). In addition, random noise was added to $(X, Z)$ coordinates with standard deviation $\delta=$ $0.0113 \mathrm{~m}$. That is, after calculating the coordinates of each point in the camera coordinate system and transferring them to the projector coordinate system, the $(X, Z)$ pairs have error with $\delta=0.0113 \mathrm{~m}$. Fig. 6(a) shows the cost function over the range of $a_{1}$ and $a_{2}$ for this dataset with increment 0.001 for both parameters. The minimum value of the cost function was $4.7357 e-05 \mathrm{~m}$ that happens at $a_{1}=-0.054$ and $a_{2}=1.183$. This shows even in the presence of relatively high amount of noise, there is only $23 \mathrm{~mm}$ error in the estimated height of water surface and 0.016 error in the slope of water surface.

Different levels of noise were added to the coordinates of those 11 rays. The above procedure was repeated to estimate the parameters of the flat water surface. The minimum value of the cost function was compared with standard deviation of errors in the input coordinates (Fig. 6 (b)). As it is expected, the higher the level of noise in coordinates, the higher the value of minimum in the cost function.

In the second synthetic dataset, linear small amplitude wave theory [21] was used to model the water surface, i.e. $Z_{i}^{w}=A \sin \left(k X_{i}^{w}-\phi\right)+B$. In this case, there were four parameters that needed to be estimated $(A, k, \phi, B)$ where $A$ is the wave amplitude, $k$ is the wavenumber $(2 \pi /$ wavelength $), \phi$ is the wave phase and $B$ is the distance from the projector to the mean water surface. Although it is clearly idealized, linear wave theory continues to be widely used for scientific and engineering calculations [39]. For this test, we set the four parameters to: $A=0.1 \mathrm{~m}, k=5\left(m^{-1}\right), \phi=\frac{-\pi}{2}$, and 


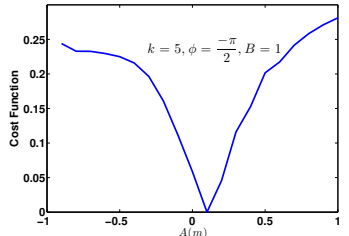

(a)

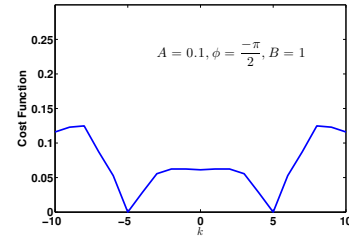

(b)

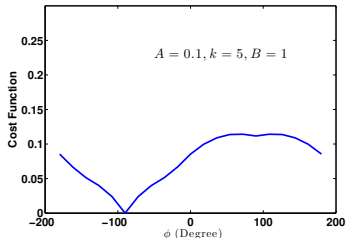

(c)

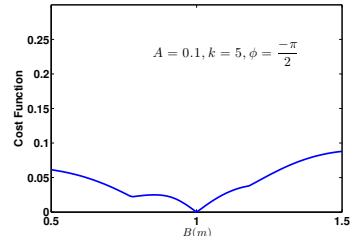

(d)

Figure 7: Cost function profiles for a synthetic sinusoidal water surface of the form $Z_{i}^{w}=$ $A \sin \left(k X_{i}^{w}-\phi\right)+B$ where $A=0.1, k=5, \phi=\frac{-\pi}{2}$, and $B=1$. Each profile represents a cross section of the four dimensional cost function along one parameter: (a)Amplitude $A$, (b)Wavenumber $k$, (c)Phase $\phi$, (d)Distance between the projector and the average water surface $B$.

$B=1$. As in the previous case, the $(X, Z)$ pairs for 11 rays were calculated using Eq.(6) to Eq.(9) . No random noise was added to the coordinates in this dataset. Noiseless data was used here as the proof of concept, we will conduct a sensitivity analysis in Section 5 to explore the effect of noise on the model. The cost function has four dimensions. Fig. 7 shows profiles of the cost function for all four parameters. In each profile, 3 parameters were fixed and set to the values corresponding to the minimum of the four dimensional cost function. Since the signal was noiseless, the estimated value of the parameters are exactly the same as their actual values. Note that for $k$ the minimum happens for two values, i.e. -5 and +5 . Plugging either of these two values into the water surface equation does not affect the shape of the water surface and it will be identical for both values. In other words, only the positive values for wavenumber could be assessed.

\subsection{Real data}

All experimental data were acquired in the Air-Sea Interaction Saltwater Tank (ASIST) at the Rosenstiel School of Marine and Atmospheric Science, University of Miami. ASIST is a state-of-the-art wind-wave tank with fully transparent acrylic walls and its primary purpose is to study various physical processes at the air-sea interface. The working section of ASIST is $15 \mathrm{~m}$ long, $1 \mathrm{~m}$ wide and $1 \mathrm{~m}$ high. For simulation of realistic ocean conditions, it is equipped with a mechanical wave generator (wave frequencies $0.25 \mathrm{~Hz}-3 \mathrm{~Hz}$, amplitudes $0-0.1 \mathrm{~m}$ ), a wind generator (wind speed in the tank's centerline 0-30 $\left(\mathrm{ms}^{-1}\right)$, a current generator (current speed 0-0.5 $\left(\mathrm{ms}^{-1}\right)$ ), and a water temperature control (5-40 C). 
The mechanical wave generator in ASIST is a flat vertical panel, moved horizontally by a hydraulic motor according to a pre-programmed trajectory. During all experiments with a wavy water surface, the shape of the motor trajectory was exclusively a sine wave with various frequencies and amplitudes.

A Canon EOS Digital Rebel XS camera was used to capture images of the distorted pseudorandom code. The camera resolution was set to $3888 \times$ 2592 pixels. An NEC MT1065 projector was used to project the calibration checkerboard and the color pattern. This projector is rated at 800:1 contrast and utilizes 3 LCDs equipped with micro lenses to increase light output. The resolution of the projector was set to its native resolution which is $1024 \times 768$ pixels (XGA). Also, the resolution of the output images from the computer was set to $1024 \times 768$ to avoid resampling of the pixels by the projector.

As a first step in 3D reconstruction, both the camera and the projector were calibrated using the method presented in Section 3.1. The projector was installed above the water surface looking downward at a height of $1.24 \mathrm{~m}$. The camera was located next to the transparent side wall of the tank. The camera-projector calibration was done while the tank was empty. Then the tank was filled with fresh water and the camera was calibrated again. The ratio of the focal length of the wet camera (filled tank) to the dry camera (empty tank) was 1.3369 which is close to the ratio of the refractive index of the water to the refractive index of the air.

Note that, it was assumed that the projector coordinate system coincides with the world coordinate system and thus all the extrinsic parameters for the projector were equal to zero. We experienced a greater variation in principal point estimation than in focal length similar to this study [40]. Thus the principal point of the projector was set to the center of the projector which is a common practice in some projector camera calibration methods [41]. Again, for a finer scale reconstruction the use of more accurate calibration methods is recommended. Fifteen images of the checkerboard at different angles were used to calibrate the camera. Also, nineteen images of the projected checkerboard on the actual checkerboard at different angles were used to calibrate the camera and the projector. All the image and the pattern coordinates used in the following experiments, were corrected for the lens distortion before applying the equations.

In the first experiment, a flat board was used as an object. A checkerboard was attached to this flat board so that the coordinates of every spot could be estimated using a calibrated camera as was described in Section 3.3. This 


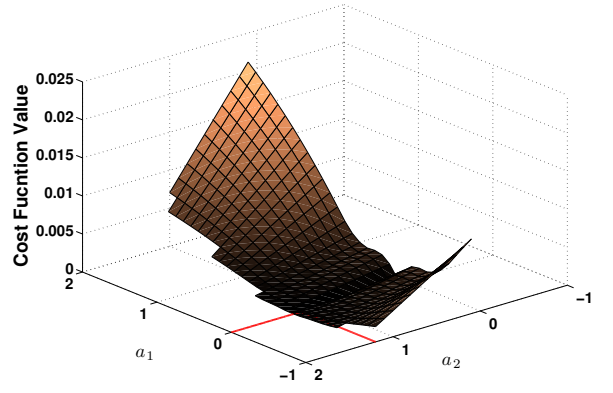

(a)

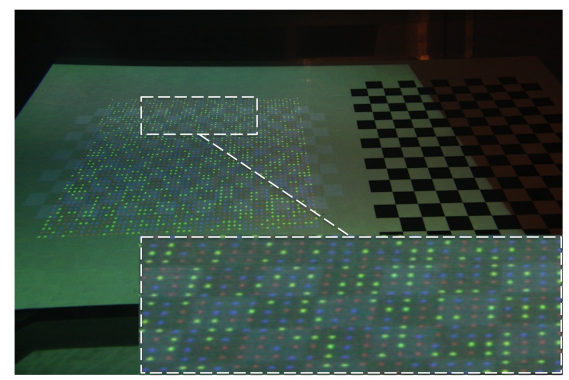

(b)

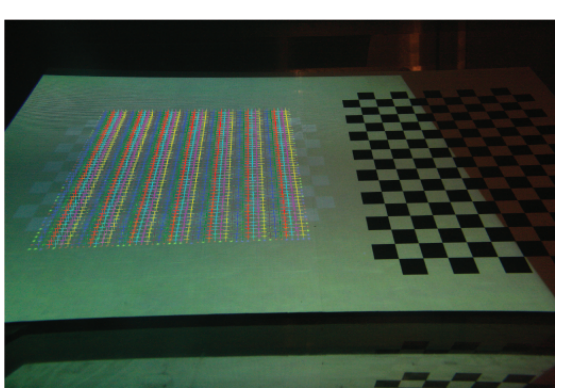

(c)

Figure 8: Results of water surface estimation for a flat water surface (no wave) (a)Cost function for $F\left(X_{i}^{w}, \mathbf{a}\right)=a_{1} x+a_{2}$ for different pairs of $a_{1}$ and $a_{2}$ with the increment of 0.1 , (b)Image of the color pattern on a flat board, (c)Extracted color pattern. The size of each square on the checkerboard is 1 inch by 1 inch.

board was placed in the tank with a slope such that it could be seen by the camera while the projector illuminated the pattern on top of it. In this experiment, the water surface was flat and no wave was generated. As can be seen in the acquired image of the pattern in Fig. 8(b), the water surface did not impose any distortion, except scaling, in the pattern. This means that if a simple linear function $F\left(X_{i}^{w}, \mathbf{a}\right)=a_{1} X_{i}^{w}+a_{2}$ represents the water surface, the slope $a_{1}$ should be zero and $a_{2}$ should be equal to the distance between the projector and the water surface. Fig. 8(a) shows the cost function for different combinations of $a_{1}$ and $a_{2}$. The increments for $a_{1}$ and $a_{2}$ were equal to 0.1 . The dark areas show lower values and the bright areas show the higher values of the cost function. Where there is no intersection between predicted refracted rays and the object (board) within a defined limit, the value of the cost function was set to infinity. As can be seen for different $a_{2}$ 's, wherever $a_{1}=0$ the minimum of the evaluation function occurs. The minimum value of all pairs of $a_{1}$ and $a_{2}$ occurs at $a_{1}=0$ and $a_{2}=1.2$ which is indicated by the intersection of two lines on Fig. 8(a). Therefor, the best representation for the water surface function would be $F\left(X_{i}^{w}, \mathbf{a}\right)=0 X+1.2$.

In the second experiment, images of the projected pattern were taken approximately every 10 minutes while the tank was filling up with a constant rate. Fig. 9(a) shows two samples of captured images. The water surface in all these images was flat and no wave was generated. With increasing height of the water surface the color pattern shrinks and this effect can be seen in the consecutive images in Fig. 9(a). Note that the camera and the projector are fixed and the distance between the projector and the water surface decreases as the tank is being filled up. Then the water surface for each image is estimated, assuming the water surface follows a flat surface model, i.e. $F\left(X_{i}^{w}, \mathbf{a}\right)=a_{1} X_{i}^{w}+a_{2}$ as in the previous experiment. The 


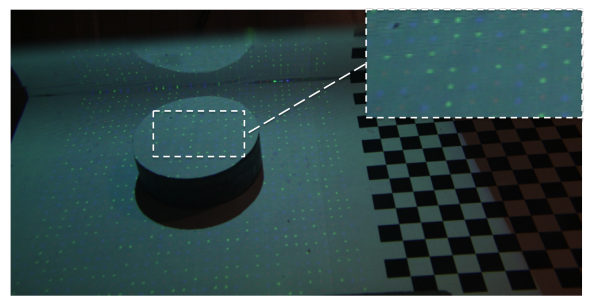

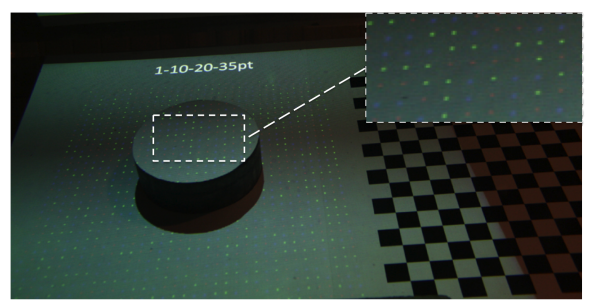

(a)

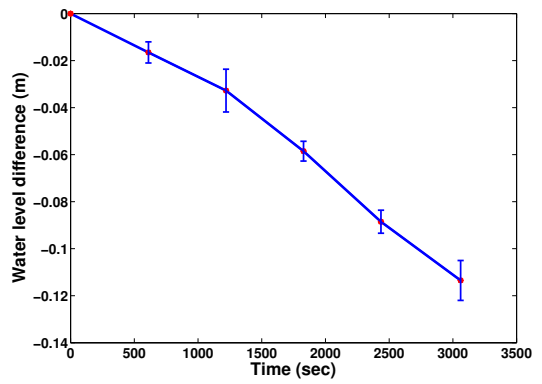

(b)

Figure 9: (a) Estimated water level differences in specific time intervals while filling up the tank with fresh water at a constant rate, (b) Two of the corresponding images of the projected pattern. The size of each square on the checkerboard is 1 inch by 1 inch.

estimated $a_{1}$ for all cases were close to zero. Fig. 9(b) shows the estimated height difference for the water surface versus time. As expected, the plot has a linear pattern and was consistent with the constant rate of filling up the tank.

In the next experiment, a wavy water surface was produced using the mechanical wave generator. It was set to generate a water surface wave using an input signal of frequency $2(\mathrm{~Hz})$, maximum voltage 1 (volt) and minimum voltage 0.5 (volt). The wave was sinusoidal and it is assumed to be modeled as $F\left(X_{i}^{w}, \mathbf{a}\right)=A \sin (k X-\phi)+B$. In this model, $A$ is amplitude, $k$ is wavenumber, $\phi$ is phase shift, and $B$ represents the distance between the projector and the mean water surface. The mechanical wave maker settings result in a wave with $k=16\left(\mathrm{~m}^{-1}\right)$ and $A=2.5(\mathrm{~cm})$. Fig. 11(a) shows the image of the pattern captured under the wavy water surface. As highlighted in the image, even under the effect of high curvature waves, small scale ripples riding on large scale mechanical waves, the color pattern is detectable and can be extracted. Although it might not be possible to extract all the projected rays, it is possible to extract a large enough number of rays in order to recover the water surface parameters. In this experiment, the sinusoidal model has four parameters and at least four rays are necessary to trigger the water surface estimation.

The cost function was calculated for the entire span of the possible solutions to find the best set of four parameters $(A, k, \phi, B)$ within defined limits for each parameter; $A \in\left[\begin{array}{ll}0 & 0.1\end{array}\right] \mathrm{m}$ with $0.02 \mathrm{~m}$ increment step, $k \in\left[\begin{array}{ll}0 & 25\end{array}\right]$ with 1 increment step, $\phi \in[-\pi \pi]$ with $\frac{\pi}{10}$ increment step, and $B \in\left[\begin{array}{ll}1 & 1.5\end{array}\right] \mathrm{m}$ with $0.02 \mathrm{~m}$ increment step. The minimum of the cost function happens at 


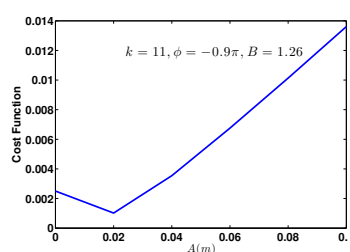

(a)

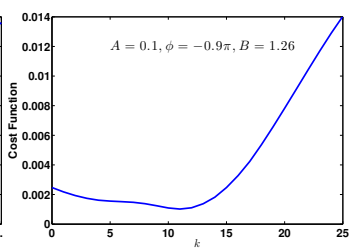

(b)

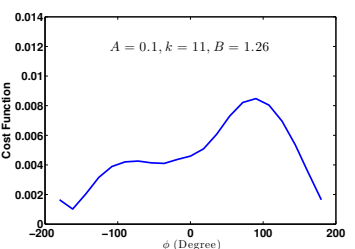

(c)

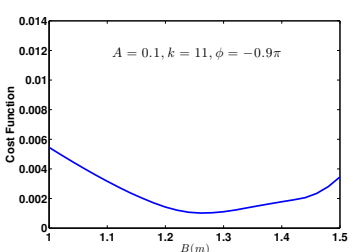

(d)

Figure 10: Cost function profiles for a wavy water surface modeled as a sinusoidal function of the form $Z_{i}^{w}=A \sin \left(k X_{i}^{w}-\phi\right)+B$. Each profile represents a cross section of the four dimensional cost function along one parameter: (a)Amplitude $A$, (b)wavenumber $k$, (c)Phase $\phi$, (d)Distance between the projector and the average water surface $B$

$A=0.02 \mathrm{~m}, k=11\left(\mathrm{~m}^{-1}\right), \phi=-2.8274$, and $B=1.26 \mathrm{~m}$. Fig. 10(a)-(d) show the profiles of the cost function for all four parameters at the minimum. The parameter $B$ which represents the distance between the projector and the average height of the water surface, is very close to the measured value, i.e. $1.24 \mathrm{~m}$. Since the area covered by the entire pattern does not contain several wavenumbers, the difference between the cost function for $k \in(0,16)$ was small (see Fig. 10 (b)). However, the cost function drastically increases for higher values of $k$. Therefore, in this experiment, by plugging values less than 16 for $k$ into the water surface model, the difference between the resulting shapes of the water surface in the region covered by the entire pattern would not be distinct. If a better estimate in the final value of $k$ is needed, the pattern has to cover a large enough area to at least encompass one full wavelength.

Assuming a sinusoidal model using linear small amplitude wave theory, the water surface parameters were calculated for seven consecutive images. The images were taken at 5 second intervals while the wave generator was creating a wavy water surface with the same characteristics. Fig. 11(a) shows two of the seven consecutive images. Ideally estimated $A, B$, and $k$ should be the same for all these images while the shift parameter $\phi$ might be different for each image. As in the previous experiment the cost functions for different combinations of parameters were calculated and the set of parameters corresponding to the minimum value of the cost function were picked as the final solution. Table 1 summarizes the final results for all these images. As expected, the parameter $A$ is equal for all seven images. Note that a $2(\mathrm{~cm})$ step size for $A$ was used in calculating the cost function and if a finer step size were used, the estimated values would be slightly different but within an 

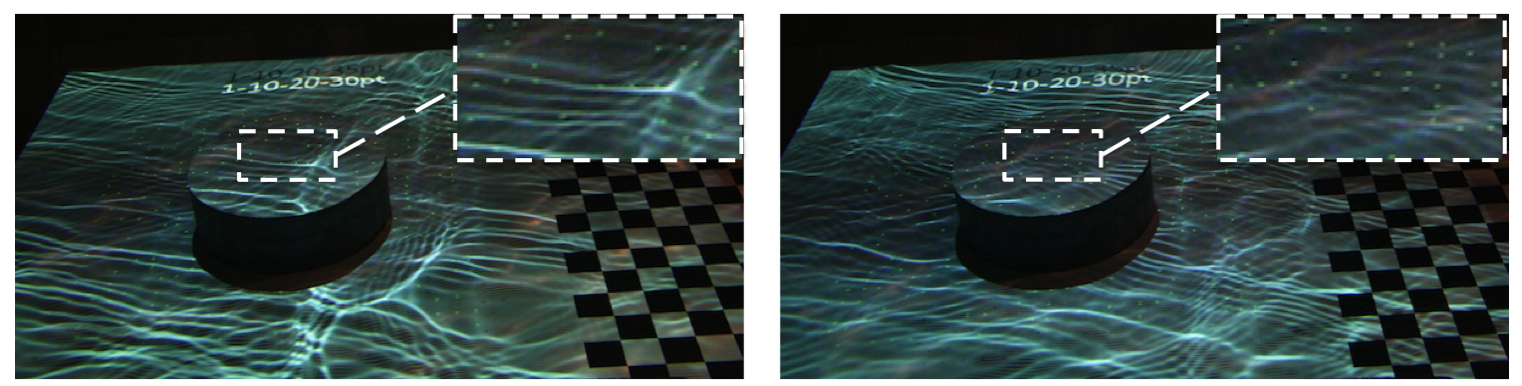

(a)

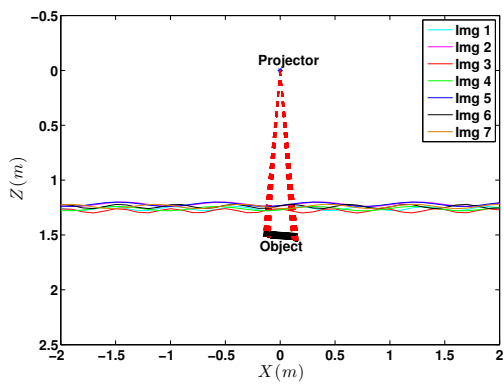

(b)

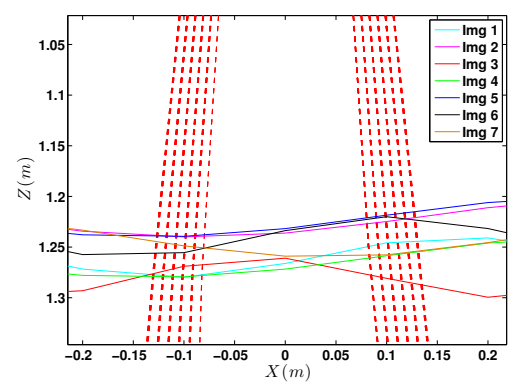

(c)

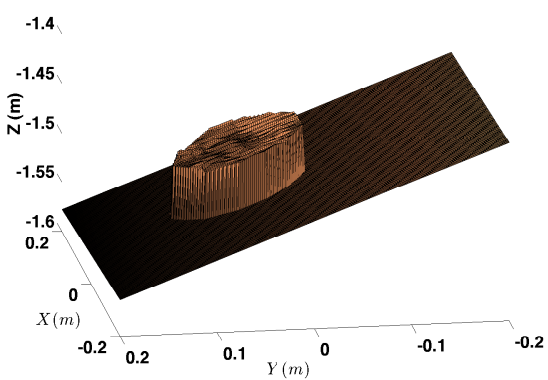

(d)

Figure 11: (a)Two samples of the seven consecutive images of a flat board and cylindrical object at the presence of wavy water surface taken by an underwater camera. The size of each square on the checkerboard is 1 inch by 1 inch. (b) Reconstructed sinusoidal water surfaces for each image with respect to the location of the projector and the object, (c) Reconstructed water surfaces are highlighted for better visual representation, (d) Reconstructed cylindrical object attached to the board using combined data from all seven images.

acceptable range. Also, the distance between the projector and the average water surface, $B$, for all images are within the expected range. As previously mentioned, since the color pattern does not contain a full wavelength of the generated wave, the value of $k$ does not affect drastically the shape of the final water surface, i.e. there is a subtle difference between the water surfaces obtained by plugging different numbers of $k<16$ into the sinusoidal water surface model. For $k>=16$ the water surface model represents one (or more than one) full wavelength within the borders of color pattern and the effect of $k$ would be more visible. Given that in all images a good estimate of $k$ was obtained. The value for shift parameter $\phi$ as expected is different for each image. Fig. 11(b) demonstrates the position of the projector, object, and the estimated water surface with respect to each other. Fig. 11(b)-(c) shows all the reconstructed water surfaces using the parameters estimated from seven images.

A better way to assess the accuracy of the proposed method is to reconstruct an object with known geometric shape and compare the reconstructed shape with the original one. For this purpose, a cylindrical object with known thickness $(0.053 \mathrm{~m})$ was attached to a board. Several images of this object 
Table 1: Estimated water surface parameters in seven images shown in Fig.11(a) using a sinusoidal model $Z=A \sin (k X-\phi)+B$ for the water surface.

\begin{tabular}{|l|lllllll|}
\hline Image Number & 1 & 2 & 3 & 4 & 5 & 6 & 7 \\
\hline$A$ & 0.02 & 0.02 & 0.02 & 0.02 & 0.02 & 0.02 & 0.02 \\
\hline$k$ & 11 & 7 & 13 & 7 & 7 & 12 & 8 \\
\hline$\phi$ & -2.8274 & -2.1991 & 1.2566 & -2.5133 & -2.5133 & 2.8274 & -1.2566 \\
\hline$B$ & 1.26 & 1.22 & 1.28 & 1.26 & 1.22 & 1.24 & 1.24 \\
\hline Average Error $(\mathbf{m})$ & 0.002 & 0.0023 & 0.0019 & 0.0016 & 0.0018 & 0.0023 & 0.0013 \\
\hline
\end{tabular}

were taken while a wavy water surface was generated (see Fig. 11(a)). The water surface was estimated for each image in the previous experiment and the results are summarized in Table 1 . The increment steps for all parameters of the sinusoidal model are the same as in the previous experiment. Then, using the estimated water surface parameters and Eq.(15) and Eq.(16), the three dimensional coordinates of the projected pattern on both the cylindrical object and the board were calculated. A plane was passed through all the points on the board. Using the $3 \mathrm{D}$ coordinates of the points on the cylindrical object and the equation of the plane, the distance from each point on top of the cylinder to the plane was calculated. This distance for all the points should be the same and equal to the thickness of the cylinder. Cumulatively, in seven images, 166 points were extracted from the pattern on top of the cylindrical object. The distances for points to the board were calculated. The average error in the distance for these points was zero and the standard deviation of error was $0.0023 \mathrm{~m}$. Higher accuracy could be achieved if the rays were thinner and sharper. However, in the presence of waves, the thiner rays could disappear and are more difficult to detect in the images. Fig. 11(d) shows the reconstructed geometric shape of the board and the attached cylindrical object using all the points obtained from the seven images. Note that the geometric shape of the cylindrical object can be reconstructed with a single image, however, in this experiment the single shot method was used in a multi-shot scheme to obtain more points on top of the object.

In order to show that this method works for non-planar objects, a spherical object (a round buoy) was cut in half and attached to the board. The radius of the buoy was $83 \mathrm{~mm}$. The color pattern was projected on this object while the wave generator was making a wavy water surface (See Fig. 12(a)). Again the water surface parameters were calculated using the projected rays on the board and eventually the coordinates of projected rays on this object. The white dots on the object shown in Fig. 12(b) illustrate the extracted rays on the surface of the spherical object. Then a sphere with $83 \mathrm{~mm}$ radius was fit to these points to measure the accuracy of the estimated 


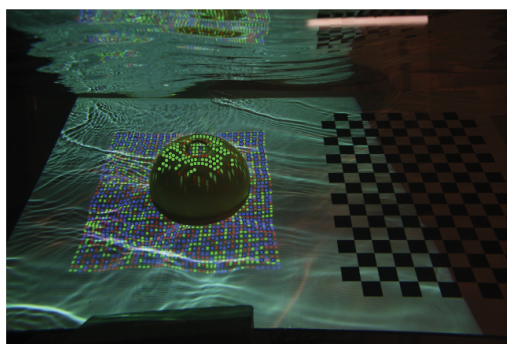

(a)

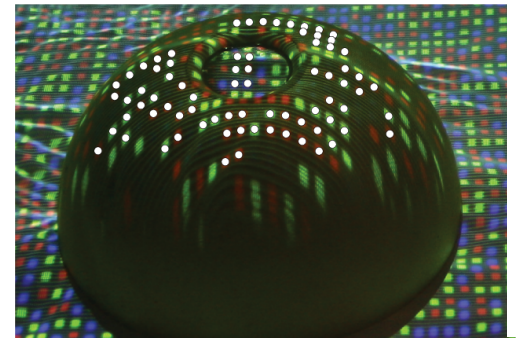

(b)

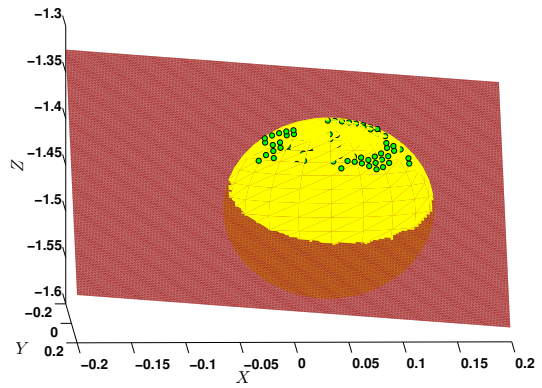

(c)

Figure 12: (a) A spherical object attached to a board illuminated by a color pattern emitted from a projector located above water. The size of each square on the checkerboard is 1 inch by 1 inch. (b) White dots depict extracted color pattern on top of the object, (c) Reconstructed spherical object attached to the board using data from one single image. Note: bigger spots are easier to extract under a wavy water surface.

coordinates. The mean of the residuals was close to zero $(2.5667 \mathrm{e}-04 \mathrm{~m})$ and their standard deviation was equal to $0.0034 \mathrm{~m}$. The reconstructed object, the extracted points on the object and the plate are shown in Fig. 12(c).

Note that even in the presence of high curvature waves, it was still possible to locate the projected rays and obtain an estimate of the water surface which was good enough to recover the $3 \mathrm{D}$ shape of underwater objects.

\section{Sensitivity analysis}

It is important to understand the behavior of the proposed method in the presence of error in the input parameters. Inevitably there would be error in the system calibration and water surface estimation. The question is how these errors will affect the accuracy of the final reconstructed object. Sensitivity analysis helps to answer this question by measuring changes in output values while changing the values of input parameters. Specifically, the effect of error in height of the estimated water surface will be assessed. Interested readers can find more details on sensitivity analysis of this method in $[42,43]$, where we assess the effect of error in slope of the estimated water surface as well as the error in the camera-projector baseline as the representative for the system calibration.

There are many approaches to sensitivity analysis. A partial derivative can be a sensitivity function for a system with well-defined equations. Statistical techniques can also be used when the model is too complex and the inputs are described with probability distributions. Since the imaging is mathematically well behaved and taking derivatives is feasible, analytic sensitivity functions will be used to evaluate the effects of error in the input parameters on the model's output. 
The following types of analytic sensitivity functions are common: Absolute, Relative, and Semi-Relative. They are all based on finding the partial derivative of a mathematical system model with respect to some parameters. The definitions of these sensitivity functions can be found in [44]. Both absolute-sensitivity and relative-sensitivity functions were used as the criterions for the assessments.

\subsection{Sensitivity of the height of water surface}

As it is shown in Fig. 1(b) and formulated in Eq.(7), the $X$ coordinate of a point is the sum of corresponding displacement in air and water, i.e. $\Delta X_{i}^{a}$ and $\Delta X_{i}^{w}$ (These formulations can be similarly applied to $Y$ coordinate as well). Error in the height of the water surface will affect both of these displacements. The absolute sensitivity function of $X$ to the height of water surface $Z_{i}^{w}$ has the following form

$$
S_{Z_{i}^{w}}^{X_{i}}=\frac{\partial X_{i}}{\partial Z_{i}^{w}}=\frac{\partial \Delta X_{i}^{a}}{\partial Z_{i}^{w}}+\frac{\partial \Delta X_{i}^{w}}{\partial Z_{i}^{w}}=\cot \left(\alpha_{i}\right)+\cot \left(\alpha_{i}+\beta_{i}\right)
$$

As can be seen, the absolute sensitivity function for the height of water surface is a function of two angles $\beta_{i}$ and $\alpha_{i}$ (See Fig. 1(b) for illustration of these two angles) and is not a function of height itself. Fig. 13 (a) depicts

$S_{Z_{i}^{w}}^{X_{i}}$ versus different $\beta_{i}$ and $\alpha_{i}$. As expected, the error in height does not have any effect on the ray at the nadir of the projector if the incidence angle is zero. In this case the effect of error in estimated height of water surface on both $\Delta X_{i}^{a}$ and $\Delta X_{i}^{w}$ is equal to zero. Remember that $\beta_{i}$ is a function of incidence angle $\theta_{i}^{a}$ and if the incidence angle is zero, the $\beta_{i}$ will also be zero. In fact, there is a (dark-blue) curve in Fig. 13 (a) which represents pairs of $\beta_{i}$ and $\alpha_{i}$ where the effect of error in height is zero. Essentially, for these pairs, the error in $\Delta X_{i}^{a}$ and $\Delta X_{i}^{w}$ due to change in height will cancel out each other and their addition is equal to zero. This curve will be distinct for each water type and will change based on the refractive index of the water.

The relative sensitivity function for the height of water surface can be obtained by multiplying the absolute sensitivity function by the height of water surface $Z_{i}^{w}$ and dividing by $X_{i}$, i.e.

$$
\begin{aligned}
\bar{S}_{Z_{i}^{w}}^{X_{i}} & =S_{Z_{i}^{w}}^{X_{i}} \frac{Z_{i}^{w}}{X_{i}} \\
& =S_{Z_{i}^{w}}^{X_{i}} \frac{Z_{i}^{w}}{\left(Z_{i}^{w}-Z_{p r o j}\right) \cot \left(\alpha_{i}\right)+\left(Z_{i}-Z_{i}^{w}\right) \cot \left(\alpha_{i}+\beta_{i}\right)}
\end{aligned}
$$




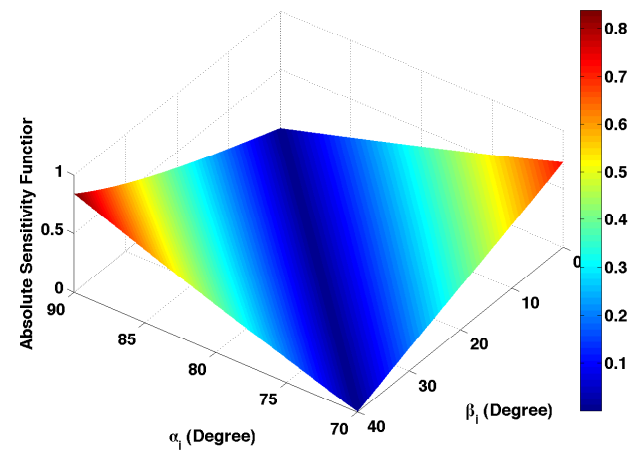

(a)

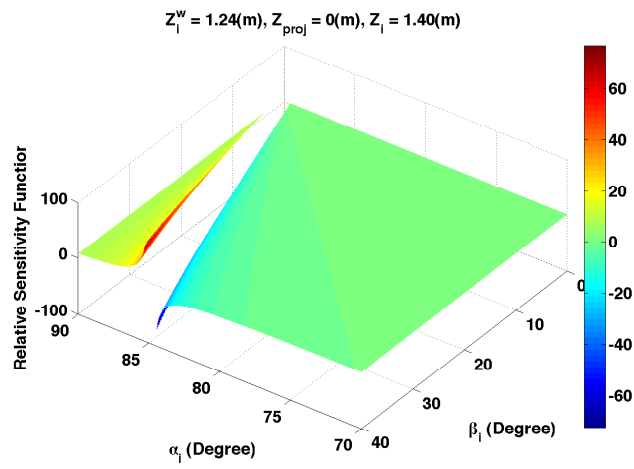

(b)

Figure 13: (a) Absolute sensitivity function and (b) relative sensitivity function for reconstructed $X$ coordinates to the height of water surface. The same parameters as in the experimental setup are used and the functions are plotted for different values of $\beta_{i}$ and $\alpha_{i}$.

As can be seen, the denominator $X_{i}$ has a similar form as absolute sensitivity function (see Eq.(17) for comparison). By looking at the absolute sensitivity function in Eq.(17), we realize that it has two components: The above-water component $\cot \left(\alpha_{i}\right)$ and the underwater $\operatorname{component} \cot \left(\alpha_{i}+\beta_{i}\right)$. The $X_{i}$ is the weighted sum of these two components and the weights are the distances in the $Z$ direction that the ray travels from the projector to the object above water $\left(Z_{i}^{w}-Z_{\text {proj }}\right)$ and underwater $\left(Z_{i}-Z_{i}^{w}\right)$. In other words, in this case, the relative sensitivity function $\bar{S}_{Z_{i}^{w}}^{X_{i}}$ is a scaled version of the absolute sensitivity function $S_{Z_{i}^{w}}^{X_{i}}$, with scale being $\left(Z_{i}^{w}\right)$, divided by the weighted sum of the two components of the absolute sensitivity function.

Fig. 13 (b) shows the relative sensitivity function $\bar{S}_{Z_{i}^{w}}^{X_{i}}$ for the experimental setup. In this case, the projector is located at $1.24 \mathrm{~m}$ above the water surface and the center of the object is at the depth equal to $16 \mathrm{~cm}$. As can be seen in Eq.(18) and illustrated in Fig.13 (b), when the denominator is zero (or close to zero), the relative sensitivity of the reconstructed $X$ coordinate to the height of the water surface is not valid. Also, note that the pairs of $\beta_{i}$ and $\alpha_{i}$ with non-valid relative sensitivity will be different for a different setup. In these cases, it is recommended to use absolute sensitivity as a criterion for sensitivity analysis.

\section{Conclusions and future work}

A method for simultaneous reconstruction of water surface and underwater structures using a camera-projector technique has been developed. The novelty of the approach is in the formulation of the reconstruction problem using a camera-projector technique while the camera is underwater and the projector is outside water. Unlike conventional structured-light methods 
where the distortion of the projected pattern is solely due to the geometric shape of the object surface, in this method, the geometric shape of the water surface and the object surface contribute to the distortion of the projected pattern.

This approach can be a basis for a wide range of underwater applications, such as mapping, archaeology, inspection of boats and shipwrecks, structures and pipes, marine biology research, and coral reef monitoring. It is specifically useful for 3D reconstruction in hazardous environments where minimal immersion in one of the media is expedient. Applying this technique to all these applications is dependent on the clarity of the water in use and the distance that the projected pattern could travel in that water. Also, depending on what percentage of error could be tolerated in an application, one should decide to use $\eta$ or $\eta^{\prime}$ in that application.

In this method, when projecting dots (color patches), their mass centers must be located and if a dot appears only partially in the image, it would be hard to accurately locate its mass center. In addition, the size of projected dots has direct impact on the final spatial resolution of reconstructed object; the smaller the dots, the better the resolution. However, decreasing the size of color dots can complicate the process of identification of these dots. One way of overcoming this issue is to use square dots and extract the corners of the color patches instead of their mass centers as was done here.

Note that this method, as most structured-light techniques, only produces partial 3D views of the scene and in order to retrieve a complete 3D model of the scene, it is necessary to merge several 3D views together.

There are many further directions beyond this work. One avenue is to extend this method to the case where both camera and the projector are in the air while the object is underwater. This would be the ideal case for non destructive testing and non invasive measurement of underwater objects since neither the camera nor the projector touch the water. However, it is more complicated because the dynamic interface must be crossed twice. Also, a more sophisticated camera model such as the one proposed in [4] can be used for modeling geometric distortions in underwater environments.

Another avenue is to explore the combination of this method with underwater image enhancement techniques such as $[45,46]$ in order to make it more suitable for environments with dominant scattering effects. An optical image taken in such a medium will be affected by significant loss of contrast and attenuation of brightness. Further, this degradation increases exponentially with distance, making it hard to capture images of the projected pattern. 
Thus, it is critical to take into account the effects of scattering while applying the proposed system in such media. Our method can also be integrated with other techniques that reconstruct the shape of water surface. Examples include a stereo imaging method suggested in [47] or the method proposed in [48] that employs light field probes.

Notice that this single-shot technique can always be translated to a multipleshot technique by expressing the color pattern over a sequence of patterns in order to achieve larger spatial resolution. One can change the size of the color dots in a hierarchical manner in a sequence of shots and achieve a higher spatial resolution. Alternatively, one can progressively shift the pattern horizontally and vertically to increase the number of 3D reconstructed points. As such, advantage is taken of the same uniquely coded feature points several times.

The water surface model can be extended to a more general case where $X$ and $Y$ coordinates are not independent. This way, the $3 \mathrm{D}$ shape of the water surface will be estimated using $X$ and $Y$ coordinates of points at the same time.

\section{Appendix A Orthogonal projection of incidence and refraction angles}

Consider Fig. 14 in which the refraction plane is defined by the incidence ray $u_{i}$, the surface normal $n$, and the refracted ray $v_{i}$, which is computed by applying Snell's law. $u_{i}$ and $v_{i}$ have equal length and thus the projections of the incoming and refracted vectors into the surface plane are in the ratio $\eta=n_{w} / n_{a}$.

Now consider the projection of the incidence and refraction rays into the $X Z$ plane, i.e. $u_{i}^{\prime}$ and $v_{i}^{\prime}$. The length of these two vectors are not equal: $\left|u_{i}^{\prime}\right|=\cos \zeta$ but $\left|v_{i}^{\prime}\right|=\cos (\delta)$, and this is the reason that these two projection vectors do not follow the Snell's law with the refractive index $\eta$. Scaling the refracted vector by $\cos (\zeta) / \cos (\delta)$ produces a Snell's law diagram for refractive index $\eta^{\prime}=\eta \cos (\delta) / \cos (\zeta)$

From the two similar triangles in the ground plane, $\eta \sin (\delta)=\sin (\zeta)$, from which it follows that $\eta^{2} \cos ^{2}(\delta)=\eta^{2}-\sin ^{2}(\zeta)$ and finally,

$$
\eta^{\prime}=\sqrt{\eta^{2}-\sin ^{2}(\zeta)} / \cos (\zeta)
$$




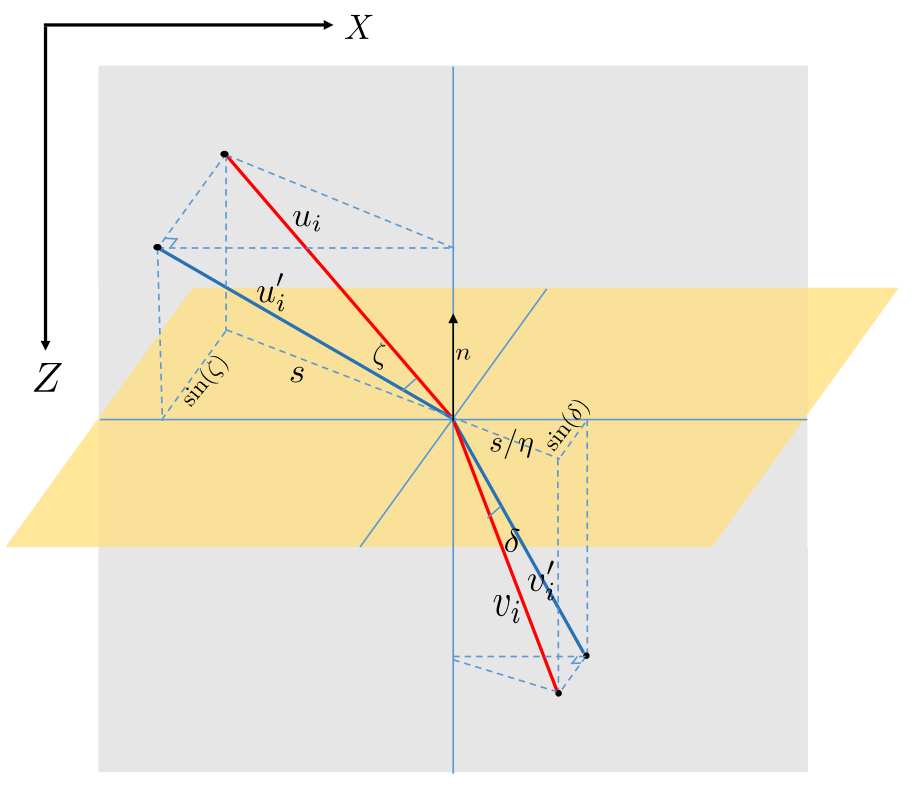

Figure 14: Incidence and refracted rays with their orthogonal projections on the $X Z$ plane

\section{Acknowledgments}

The authors would like to thank Mike Rebozo for assisting us to set up all the experiments in the lab. This work was supported by the U.S. National Science Foundation, GEO/ATM Physical and Dynamical Meteorology Program, Grant AGS0933942. This research paper was made possible, in part by a grant from BP/The Gulf of Mexico Research Initiative .

\section{References}

[1] R. Kotowski, Phototriangulation in multi-media photogrammetry, in: ISPRS Archives Volume XXVII Part B5, 1988, pp. 324-334.

[2] J. Höhle, Reconstruction of the underwater object, Journal of Photogrammetric Engineering and Remote Sensing 37 (1971) 948-954.

[3] F. Bruno, G. Bianco, M. Muzzupappa, S. Barone, A. Razionale, Experimentation of structured light and stereo vision for underwater $3 \mathrm{~d}$ reconstruction, ISPRS Journal of Photogrammetry and Remote Sensing 66 (2011) $508-518$. 
[4] G. Telem, S. Filin, Photogrammetric modeling of underwater environments, ISPRS Journal of Photogrammetry and Remote Sensing 65 (2010) $433-444$.

[5] F. Remondino, S. El-Hakim, Image-based 3d modeling: A review, The Photogrammetric Record 21 (2006) 269-291.

[6] T. Luhmann, S. Robson, S. Kyle, J. Boehm, Close Range Photogrammetry and 3D Imaging, Walter De Gruyter Inc, 2013.

[7] R. Szeliski, Computer Vision: Algorithms and Applications, Texts in Computer Science, Springer, 2010.

[8] I. Ishii, K. Yamamoto, K. Doi, T. Tsuji, High-speed 3d image acquisition using coded structured light projection, in: IEEE RSJ International Conference on Intelligent Robots and Systems, 2007, pp. 925-930.

[9] K. Sato, S. Inokuchi, Range-imaging system utilizing nematic liquid crystal mask, in: IEEE International Conference on Computer Vision, 1987, pp. 657-661.

[10] R. Valkenburg, A. McIvor, Accurate 3d measurement using a structured light system, Image and Vision Computing 16 (1996) 99-110.

[11] P. S. Huang, S. Zhang, Fast three-step phase-shifting algorithm, Applied Optics 45 (2006) 5086-5091.

[12] Z. J. Geng, Rainbow three-dimensional camera: new concept of highspeed three-dimensional vision systems, Optical Engineering 35 (1996) 376-383.

[13] P. Payeur, D. Desjardins, Structured light stereoscopic imaging with dynamic pseudo-random patterns, Image Analysis and Recognition, Lecture Notes in Computer Science 5627 (2009) 687-696.

[14] S. Fernandez, J. Salvi, T. Pribanic, Absolute phase mapping for one-shot dense pattern projection, in: PROCAMS10, 2010, pp. 64-71.

[15] M. Maruyama, S. Abe, Range sensing by projecting multiple slits with random cuts, IEEE Transactions on Pattern Analysis and Machine Intelligence 15 (1993) 647-651. 
[16] A. Ulusoy, F. Calakli, G. Taubin, One-shot scanning using de bruijn spaced grids, in: 3DIM, IEEE, IEEE, 2009, pp. 1786-1792.

[17] R. Sagawa, H. Kawasaki, S. Kiyota, R. Furukawa, Dense one-shot 3d reconstruction by detecting continuous regions with parallel line projection, in: Proceedings of the 2011 International Conference on Computer Vision, ICCV '11, IEEE Computer Society, Washington, DC, USA, 2011, pp. 1911-1918.

[18] J. Geng, Structured-light 3d surface imaging: a tutorial, Advances in Optics and Photonics 3 (2011) 128-160.

[19] J. Salvi, J. Pags, J. Batlle, Pattern codification strategies in structured light systems, Pattern Recognition 37 (2004) 827-849.

[20] D. Lay, Linear Algebra and Its Applications, Pearson Education, 2011.

[21] G. B. Airy, On tides and waves, Encyclopaedia Metropolitania 5 (1845) 241-396.

[22] D. C. Brown, Close-range camera calibration, Photogrammetric Engineering 37 (1971) 855-866.

[23] A. Y. I. Aziz, H. M. Karara, Direct linear transformation into object space coordinates in close-range photogrammetry, in: Proceeding of the Symposium on Close-Range Photogrammetry, Urbana, Illinois, 1971, pp. 1-18.

[24] C. Fraser, Digital camera self-calibration, ISPRS Journal of Photogrammetry and Remote Sensing 52 (1997) 149-159.

[25] C. S. Fraser, A. S. S. Ajlouni, Zoom-Dependent Camera Calibration in Digital Close-Range Photogrammetry, Photogrammetric Engineering and Remote Sensing 72 (2006) 1017-1026.

[26] T. Clarke, J. Fryer, The Development of Camera Calibration Methods and Models, The Photogrammetric Record 16 (1998) 51-66.

[27] M. R. Shortis, S. ROBSON, H. A. BEYER, Principal point behaviour and calibration parameter models for kodak dcs cameras, Photogrammetric Record 16 (1998) 165-186. 
[28] R. Tsai, A versatile camera calibration technique for high-accuracy 3d machine vision metrology using off-the-shelf tv cameras and lenses, IEEE Journal of Robotics and Automation 3 (1987) 323 -344.

[29] Z. Zhang, A flexible new technique for camera calibration, IEEE Transactions on Pattern Analysis and Machine Intelligence (2000) 1330-1334.

[30] J. Heikkila, O. Silven, A four-step camera calibration procedure with implicit image correction, in: IEEE Computer Society Conference on Computer Vision and Pattern Recognition, 1997, pp. 1106 -1112.

[31] F. Remondino, C. Fraser, Digital camera calibration methods: Considerations and comparisons, in: International archives of photogrammetry and remote sensing, volume 36, ISPRS, 2006, pp. 266-272.

[32] J. Salvi, X. Armangu, J. Batlle, A comparative review of camera calibrating methods with accuracy evaluation, Pattern Recognition 35 (2002) 1617 - 1635.

[33] Z. Zhang, Flexible camera calibration by viewing a plane from unknown orientations, in: The Proceedings of IEEE International Conference on Computer Vision, volume 1, 1999, pp. $666-673$. doi:10.1109/ICCV.1999.791289.

[34] J.-Y. Bouguet, Camera calibration toolbox for matlab, 2000. URL: http://www.vision.caltech.edu/bouguetj/calib_doc/.

[35] M. Kimura, M. Mochimaru, T. Kanade, Projector calibration using arbitrary planes and calibrated camera., in: IEEE Conference on Computer Vision and Pattern Recognition, IEEE Computer Society, 2007.

[36] G. Falcao, N. Hurtos, J. Massich, D. Fofi, Projector-camera calibration toolbox, 2009. URL: http://code.google.com/p/procamcalib.

[37] D. Desjardins, P. Payeur, Dense stereo range sensing with marching pseudo-random patterns, in: Proceedings of the Fourth Canadian Conference on Computer and Robot Vision, IEEE Computer Society, Washington, DC, USA, 2007, pp. 216-226.

[38] R. Hartley, A. Zisserman, Multiple View Geometry in Computer Vision, Cambridge books online, Cambridge University Press, 2003. 
[39] M. E. McCormick, Ocean Engineering Mechanics: with Applications, Cambridge University Press, Cambridge New York, 2010.

[40] R. Raskar, P. Beardsley, A self-correcting projector, in: Proceedings of the 2001 IEEE Computer Society Conference on Computer Vision and Pattern Recognition, volume 2, 2001, pp. II-504-II-508 vol.2.

[41] T. Okatani, K. Deguchi, Autocalibration of a projector-camera system, IEEE Transactions on Pattern Analysis and Machine Intelligence 27 (2005) 1845-1855.

[42] A. Sarafraz, B. K. Haus, Performance assessment of an underwater topographic reconstruction method, in: ASPRS 2013 Annual Conference, Baltimore, USA, 2013.

[43] A. Sarafraz, Topographic Reconstruction Through a Wavy Interface Using a Structure Light Technique, Ph.D. thesis, University of Miami, 2012 .

[44] E. D. Smith, F. Szidarovszky, W. J. Karnavas, A. T. Bahill, Sensitivity analysis , a powerful system validation technique, The Open Cybernetics and Systemics Journal 2 (2008) 39-56.

[45] S. G. Narasimhan, S. K. Nayar, B. S. B. Sun, S. J. Koppal, Structured light in scattering media, in: 10th IEEE International Conference on Computer Vision, volume 1, IEEE, 2005, pp. 420-427.

[46] A. Sarafraz, S. Negahdaripour, Y. Y. Schechner, Enhancing images in scattering media utilizing stereovision and polarization (2009).

[47] N. J. Morris, K. N. Kutulakos, Dynamic refraction stereo, IEEE Transactions on Pattern Analysis and Machine Intelligence 33 (2011) 15181531.

[48] G. Wetzstein, D. Roodnick, W. Heidrich, R. Raskar, Refractive shape from light field distortion., in: ICCV, IEEE, 2011, pp. 1180-1186. 